\title{
Two-year data comparison of ab interno trabeculectomy and trabecular bypass stenting using Exact Matching
}

Hamed Esfandiari, ${ }^{1}$ Kenneth Taubenslag, ${ }^{1}$ Priyal Shah, ${ }^{1}$ Swati Goyal, ${ }^{2}$ Adam J. Weiner, ${ }^{3}$ Melissa L. Severson, ${ }^{4}$ Asher Weiner, ${ }^{4}$ Davinder S. Grover, ${ }^{5}$ Igor I. Bussel, ${ }^{1}$ Nils A. Loewen ${ }^{1}$

${ }^{1}$ Department of Ophthalmology, School of Medicine, University of Pittsburgh, Pittsburgh, Pennsylvania, United States

${ }^{2}$ Eye Q Superspeciality Eye Hospital, Gurgaon, India.

${ }^{3}$ Beaumont Eye Institute, Oakland University William Beaumont School of Medicine, Royal Oak, MI, USA. ${ }^{4}$ Department of Ophthalmology and Ira G. Ross Eye Institute, Jacobs School of Medicine and Biomedical Sciences, University at Buffalo/State University of New York (SUNY), Buffalo, NY.

${ }^{5}$ Glaucoma Associates of Texas, Dallas, TX.

Correspondence: Nils A. Loewen, MD, PhD University of Pittsburgh, School of Medicine Department of Ophthalmology 203 Lothrop St. Pittsburgh, PA 15213

Email: loewen.nils@gmail.com

Phone: 412-944-2554 Fax: 412-647-5119 


\section{Abstract}

Purpose: To create a highly balanced comparison of ab interno trabeculectomy (Trabectome, AIT) and trabecular bypass stenting (iStent, TBS).

Setting: Eye and Ear Institute, Pittsburgh, Ross Eye Institute, Buffalo, and Glaucoma Associates of Texas, Dallas, USA.

Design: Retrospective Exact Matching analysis.

Methods: AIT and TBS patients were included from three large glaucoma practices. The primary outcome measure was the unmedicated IOP $\leq 21 \mathrm{mmHg}$ at 2-year follow-up visit. A secondary measure was unmedicated IOP reduction $\geq 20 \%$ at 2 years. Patients were matched by baseline IOP, the number of glaucoma medications and glaucoma type using Exact Matching and by age using Nearest Neighbor matching. Patients without a close match were excluded. All surgeries were combined with phacoemulsification.

Results: 154 AIT and 110 TBS eyes were analyzed. 48 AIT patients were exactly matched to 48 TBS patients. Both groups had a baseline IOP of $15.3 \pm 3.1 \mathrm{mmHg}$. At 24 months, the mean IOP was $13.9 \pm 3.3$ for AIT versus $16.8 \pm 2.8 \mathrm{mmHg}$ for TBS $(p=0.04)$, while the number of medications was $0.7 \pm 1.0$ for AIT versus $1.7 \pm 1.2$ for TBS ( $p=0.04)$. The proportion of subjects achieving IOP $\leq 21 \mathrm{mmHg}$ without medications at 24 months was $53 \%$ in AIT versus $16.6 \%$ in TBS $(P<0.05)$. At 24 -month follow up $17.6 \%$ of patients in AIT had $\geq 20 \%$ IOP reduction without medication versus no patient in TBS.

Conclusions: An Exact Matching comparison of AIT and TBS demonstrated greater IOP reduction with fewer medications in AIT.

Keywords: ab interno trabeculectomy, trabectome surgery, iStent, phacoemulsification, glaucoma surgery. 


\section{Introduction}

Microincisional glaucoma surgeries (MIGS) are often chosen as a first procedure over traditional surgeries (trabeculectomy, tube shunt) due to their favorable safety profile with approximately only $1 / 20$ th of serious complications. ${ }^{1}$ MIGS that enhance the conventional outflow ${ }^{2}$ are limited by the episcleral venous pressure of about $8 \mathrm{mmHg}$ while suprachoroidal or subconjunctival shunts can cause hypotony. ${ }^{3}$ In the present study, we compared ab interno trabeculectomy (AIT) with the Trabectome (NeoMedix Corp, Tustin, CA), to trabecular bypass stenting (TBS) with the iStent (Glaukos Corp, San Clemente, CA). Both surgeries can be combined with cataract surgery and were so in all cases here. The Trabectome was introduced in 2004 for pediatric and adult open-angle glaucoma. A key feature is its drag free trabecular meshwork (TM) ablation over up to 180 degrees by plasma generated in a confined space at the tip. In contrast, the iStent was introduced in 2012 for treatment of open-angle glaucoma in combination with cataract surgery. This trabecular bypass stent is made out of titanium and inserted through the TM into Schlemm's canal under gonioscopic view after the anterior chamber is inflated with viscoelastic. Recent reports described a progressive tissue response to the TBS akin to fibrosis ${ }^{4}$ and a biofilm deposition. ${ }^{5}$

Two retrospective studies assessed the relative safety and efficacy of the two procedures ${ }^{6,7}$ but compared two stents to Trabectome surgery ${ }^{6}$ or had relatively unequal baseline parameters. ${ }^{7}$ Although a randomized controlled trial typically yields the highest quality evidence, recent statistical matching strategies ${ }^{8}$ can produce a highly balanced comparison of interventions utilizing extant data. We used Coarsened Exact Matching in studies where the data archetypes and distribution required coarsening properties into bins due to uneven treatment groups, for instance, AIT with versus without cataract surgery ${ }^{9}$ and Propensity Score Matching for tube shunt and AIT comparisons where treatment cohorts were more dissimilar and the number of potential confounding factors large. ${ }^{10,11}$ In the present study, AIT and TBS treatment groups had very similar data and indications, lending itself to Exact Matching of the two key parameters, intraocular pressure (IOP) and the number of medications to achieve this IOP.

The purpose of this study was to use Exact Matching to discover treatment effect differences that are easily missed when mere group averages are compared as done in prior studies comparing AIT and TBS. ${ }^{6,7}$ We hypothesized that the presence of a chronic titanium foreign body (TBS) in the TM, a tissue rich with cells that can phagocytose ${ }^{12,13}$ and present antigens ${ }^{14,15}$ would result in a different effectiveness over time compared to TM ablation by AIT. 


\section{Methods}

\section{Study design}

Data for this study were collected with approval from the Institutional Review Board of the University of Pittsburgh (PRO14100026) in accordance with the Declaration of Helsinki and the Health Insurance Portability and Accountability Act. Patients who underwent either AIT or TBS between January of 2008 and December of 2015 were identified by Current Procedural Terminology code. Exclusion criteria included age less than 20 years, neovascular glaucoma, uveitic glaucoma with uncontrolled uveitis, and prior ocular surgery. Baseline visits were reviewed for the demographic information, glaucoma type and stage, preoperative intraocular pressure (IOP) and number of glaucoma medications, and best corrected visual acuity (BCVA). For all postoperative visits, IOP, number of glaucoma medications, and BCVA were also recorded. Glaucoma stage at the time of surgery was assessed in standard fashion ${ }^{16}$ by the managing glaucoma specialist based on the most recent Humphrey visual field exams (Zeiss, Jena, Germany).

The primary efficacy outcome measure was the proportion of patients with IOP $\leq 21 \mathrm{mmHg}$ without ocular hypotensive medication at 2 years follow-up. The proportion of patients with a $\geq 20 \%$ reduction in IOP from baseline without medication was selected as the secondary efficacy outcome.

The indication for surgery (including the decision of which surgery to proceed with) was determined by the individual surgeon when the IOP was above the targeted IOP on maximally tolerated medical treatment or stable IOP with a desire to reduce glaucoma medications. Procedures were performed by the same group of surgeons on comparable patient populations, and both procedures were combined with phacoemulsification and intraocular lens implantation in all cases. A decision to resume medications or to reoperate was also made by the individual surgeon.

\section{Statistics}

To describe data, frequency, percentage, mean $\pm S D$, median, and range were used. Demographics were compared by the Mann-Whitney $U$ test and chi-squared test for continuous and categorical variables, respectively. Using the Matchlt package in $\mathrm{R}$, groups were then matched $\mathrm{R}$ by Exact Matching based on baseline IOP, glaucoma medications and glaucoma type and by age using Nearest Neighbor Matching. ${ }^{17}$ Exact Matching matched each unit in group 1 (AIT) to all possible control units in group 2 (TBS) with exactly the same values on all the covariates, while Nearest Neighbor Matching selected the best matches based on distance to the value in group 1. Univariate linear regression was used to examine IOP reduction after surgery. Statistically significant variables were included in the final multivariate regression model. A p-value 
of less than 0.05 was considered statistically significant. Continuous variables were expressed as mean $\pm S D$. Pre-match data was also analyzed. All statistics were performed using R.

\section{Surgical technique}

AIT and TBS were performed in combination with phacoemulsification in all cases. Angle surgeries were performed first, followed by phacoemulsification and lens implantation. AIT was performed as described before. ${ }^{18}$ Briefly, a $1.6 \mathrm{~mm}$ uniplanar, temporal clear corneal incision was created. Under direct gonioscopic view, the tip of the handpiece was inserted into Schlemm's canal for counterclockwise TM ablation followed by counterclockwise ablation maximizing the extent to the surgeon's ability (between 120 to $\left.180^{\circ}\right)$. Ablation was initiated with the power set to $0.8 \mathrm{~mW}$ and increased as necessary. The handpiece was withdrawn from the anterior chamber, and the incision was hydrated to seal.

TBS were implanted as described before. ${ }^{19}$ Briefly, after creating a temporal clear corneal incision, an ophthalmic viscoelastic device was injected into the anterior chamber to widen the nasal angle. By maintaining a low to moderate pressure reflux of blood into Schlemm's canal was permitted to help surgeons identify the angle's landmarks and collector channel openings. The inserter was brought across the anterior chamber toward the nasal angle under direct gonioscopic view. The stent was inserted through the TM, and into Schlemm's canal, then released and the inserter retracted and the viscoelastic removed. Three surgeons performed these surgeries and followed the same standard techniques. ${ }^{18,19}$

In both AIT and TBS, postoperative treatment comprised a combination of a topical antibiotic for one week and a steroid tapered over 4 weeks. In AIT, pilocarpine was used four times a day for one month followed by three times a day for a second month, a practice only recently found to be unnecessary. ${ }^{20}$ Glaucoma medications were stopped at the day of surgery and restarted postoperatively as needed.

\section{Results}

Out of 154 AIT and 110 TBS patients, 48 AIT were matched to 48 TBS using Exact Matching. There was no significant difference in IOP, the number of IOP-lowering medications, glaucoma type, or VF loss between groups ( $p>0.05$ ). Table 1 shows the baseline characteristics of each group. This pre-matched data showed no difference in the number of medications, type of glaucoma, or visual field indices. (Supplementary Table 1). In contrast, the pre-match IOP was higher in AIT than in TBS $(17.6 \pm 5.2$ versus $15.5 \pm 4.1 \mathrm{mmHg}$, respectively; $\mathrm{P}<0.01)$. Both AIT and TBS were combined with cataract extraction.

Matched groups had the same preoperative IOP of $15.3 \pm 3.1 \mathrm{mmHg}$. The mean IOP at 1 month was 
12.4 $\pm 3.5 \mathrm{mmHg}$ for AIT versus $14.5 \pm 3.9 \mathrm{mmHg}$ for TBS $(p=0.01), 13.4 \pm 3.8 \mathrm{mmHg}$ for AIT versus $14.3 \pm 2.6$ $\mathrm{mmHg}$ for TBS ( $\mathrm{p}=0.04$ ) at 12 months (Table 2). At 24 months, the mean IOP was $13.9 \pm 3.3 \mathrm{mmHg}$ for AIT versus $16.8 \pm 2.8 \mathrm{mmHg}$ for TBS ( $\mathrm{p}=0.04$, Figure 1$)$. The number of IOP-lowering medications at baseline was 1.3 \pm 1.0 for AIT versus $1.3 \pm 1.0$ for TBS. At twelve months, it was $0.7 \pm 1.1$ for AIT versus $0.9 \pm 1.0$ for TBS $(p=0.19$, Table 2$)$. At 24 months, the number of medications was $0.7 \pm 1.0$ for AIT versus $1.7 \pm 1.2$ for TBS $(p=0.04$, Figure 1). The pre-matched data analysis showed the same trend of lower IOP in AIT compared to TBS (Supplementary Table 2).

The proportion of subjects achieving the primary efficacy outcome of an IOP $\leq 21 \mathrm{mmHg}$ without ocular hypotensive medications at 24 months was greater in AIT with 53\%, than in TBS with 16.6\% (P< 0.05 , Figure 2 ). The proportion of patients achieving the secondary efficacy endpoint of IOP reduction $\geq$ $20 \%$ without an ocular hypotensive medication at 12 months was also significantly greater in AIT where it was $27.6 \%$ compared to TBS where it was $8.8 \%(P<0.05)$. At 24 -month follow up, $17.6 \%$ of patients in AIT had a more than 20\% IOP reduction without medication while no patient in TBS achieved this goal at 24month follow-up (Figure 2). The efficacy of AIT in both the primary and secondary endpoints was consistently greater than TBS alone at all time points, with the treatment difference at 6 months continuing throughout the balance of the 24-month follow-up period (Figure 2). The cumulative probability of success in AIT, defined as IOP $\leq 21 \mathrm{mmHg}$ and $\geq 20 \%$ IOP reduction was $58 \%$ at 12 months and $50 \%$ at 24 months. The corresponding numbers in TBS were $43 \%$ at 12 months and $30 \%$ at 24 months (Figure 3). Broadening the success definition to either IOP $\leq 21$ or $\geq 20 \%$ (instead of IOP $\leq 21$ and $\geq 20 \%$ ) to reflect the fact that many patients did not require a lower IOP because they had stable glaucoma, increased the success rate to 98\% and 95\% in AIT and TBS, respectively (Figure 4).

The concurrent cataract surgery, performed in all patients, improved BCVA at 24 months from $0.48 \pm 0.17 \log M A R$ to $0.10 \pm 0.14 \log M A R(p<0.001)$ in AIT and from $0.44 \pm 0.25 \log M A R$ to $0.08 \pm 0.10$ $\log$ MAR $(p<0.001)$ in TBS. There was no significant difference in BCVA between the two groups at any time $(p>0.05)$. No intraoperative and postoperative complications were observed but a common occurrence was a postoperative micro- or small layered hyphema on postoperative day 1 in $35 \%$ of AIT and 0.8\% of TBS $(P=0.01)$. No vision-threatening complications including choroidal effusion, sustained hypotony, choroidal hemorrhage, or infection occurred. 


\section{Discussion}

Ab interno angle surgeries like AIT and TBS have a proven track record of efficacy and safety ${ }^{2,10,21}$ with relatively similar outcomes. However, the recent demonstration of fibrosis ${ }^{4}$ and biofilm deposition ${ }^{5}$ in TBS specimens is concerning for a declining function caused by a metal foreign body that is absent in AIT. The better performance of AIT compared to the weaning effect of TBS detected by Exact Matching might be directly related to this and indicate a progressive failure. Such a difference gets likely missed when group averages are compared as is often done in retrospective studies that often suffer from inadvertent bias. In contrast, Exact Matching created highly equal pairs with an identical baseline of IOP and medications, key parameters describing the preoperative glaucoma treatment. Important distinctions between AIT and TBS are that the extensive removal of TM in AIT allows for a broader access to the outflow system. ${ }^{22}$ On one hand, the TBS is surrounded by the TM, a reactive tissue, ${ }^{12-15}$ yet depends on the patency of its small lumen. On the other hand, no foreign material remains in the eye in AIT but the access to the outer wall of Schlemm's canal and collector channel orifices are unguarded and can become blocked by iris ${ }^{23}$ or proliferating cells.

In our study, the relative IOP reduction by AIT of about $13 \%$ was lower than reported before because of an already low baseline IOP and a dual indication for removing a visually significant cataract as well as reducing the pressure or drops. In contrast, patients with an IOP reduction as the sole indication typically have a higher preoperative IOP yet still reach the same postoperative pressure. ${ }^{24}$ In theory, removal or bypassing the TM should decrease the IOP to the $8 \mathrm{mmHg}$ present in the receiving episcleral veins $^{25}$ but practically, both TBS and AIT reach a postoperative IOP of approximately $16 \mathrm{mmHg}$ instead. ${ }^{1,6,7}$ This discrepancy suggests an unidentified post-TM outflow resistance that may be more significant in glaucoma than in healthy eyes. ${ }^{26-28}$ In our study, AIT led to a $50 \%$ reduction of glaucoma medications at the 2-year follow-up despite the low preoperative IOP. In TBS, however, medications had to be increased gradually starting at 1 month but could still not prevent an IOP increase over time.

Although prior studies suggested similar results of AIT and TBS, ${ }^{7,29}$ data from different patient populations naturally suffer from bias as is evident in our own pre-match data. Our AIT patients had a higher IOP than TBS before matching suggesting that AIT was a surgeon preference in individuals with higher pressure. AIT lowered the IOP by approximately $13 \%$ to $13.9 \mathrm{~mm} \mathrm{Hg}$ and decreased the number of medications by less than one while maintaining a low rate of serious complications. As seen in previous reports, ${ }^{1,30}$ IOP and medications needed to achieve this IOP remained mostly stable. In this study, we found 
a significant treatment effect in favor of AIT, lowering IOP with fewer medications compared to TBS. At 24 months postoperatively, 37\% more AIT patients achieved the commonly used IOP endpoint of equal to or less than $21 \mathrm{mmHg} .{ }^{31}$ Although individual targets vary, complete surgical success for mild to moderate glaucoma is often defined as IOP $\leq 21 \mathrm{mmHg}$ without glaucoma medications. ${ }^{10,32}$ A $20 \%$ IOP reduction is defined as a "level A" target recommendation by the American Academy of Ophthalmology for glaucoma. ${ }^{33}$ The proportion of AIT subjects achieving this with no medications exceeded that of TBS subjects by almost 18\%. Overall, more subjects had greater IOP lowering benefit from AIT than TBS. No patient in TBS achieved this endpoint of success at 24-month follow-up.

Limitations of our study are that not only TBS plus phacoemulsification but also phacoemulsification on its own lowers IOP. ${ }^{34}$ This raises the question whether the IOP reduction by TBS in our study is inadvertently helped by this additional effect. ${ }^{35}$ The same would not apply to AIT where same session cataract surgery has no significant contributory effect ${ }^{9,36}$ presumably because less TM remains in the way of aqueous flow ${ }^{22}$ that could benefit from a cataract surgery-mediated, trabeculoplasty-like mechanism. In contrast, the single lumen access to Schlemm's canal of TBS limits the circumferential flow to approximately 30 degrees on each side. ${ }^{22}$ TBS strategies have now evolved to attempt implantation near presumed collector channel openings in hopes for better flow ${ }^{37}$ and the implant itself has been redesigned into two smaller stents that can be delivered to different sites to make it more effective. ${ }^{38}$

Additional limitations of our study are that Exact Matching must exclude highly different patient data sets to mimic randomization and reduce bias of confounding variables. Doing so resulted in patients with a low baseline IOP. While this allowed us to discover an adverse IOP increase, patients with a high preoperative IOP might respond differently to TBS implantation but were not available in this data set. Another shortcoming was that AIT was put at a disadvantage: the average age was five years lower than in TBS and age is known to correlate with a diminished IOP reduction. ${ }^{36}$ The percentage of African-American patients was higher in AIT than in TBS and it is possible that fibrosis that contributed to the TBS's decline could have been more pronounced with even ethnicities in both groups.

In summary, Exact Matching of AIT and TBS data resulted in a highly-balanced comparison. AIT surpassed TBS in IOP reduction and number of glaucoma medications needed postoperatively. 


\section{What was known:}

1- Trabectome-mediated ab interno trabeculectomy combined with phacoemulsification or on its own effectively reduces IOP.

2- Trabecular bypass stenting using the iStent combined with phacoemulsification effectively reduces IOP but recent studies indicate a chronic tissue response to the implant.

\section{What this paper adds:}

Over two years follow-up, Trabectome-mediated ab interno trabeculectomy combined with cataract surgery resulted in a greater reduction of IOP and glaucoma medications than trabecular bypass stenting using the iStent.

\section{Funding}

We acknowledge support from The Initiative to Cure Glaucoma, The Eye and Ear Foundation of Pittsburgh; NIH CORE Grant P30 EY08098 to the Department of Ophthalmology; from an unrestricted grant from Research to Prevent Blindness, New York, NY.

\section{Conflict of interest}

NAL and AW have received honoraria for Trabectome wet labs and lectures from Neomedix Corp. DSG: Consultant (Allergan, New World Medical, Reichert, Shire); Lecturer (Allergan, Bausch + Lomb, New World Medical, Reichert); Medical Advisory Board (MicroOptx)HE, SG, PS: none, IB,KT, HE: None All procedures performed in studies involving human participants were in accordance with the ethical standards of the institutional and/or national research committee and with the 1964 Helsinki declaration and its later amendments or comparable ethical standards. 


\section{References}

1. Kaplowitz K, Bussel II, Honkanen R, Schuman JS, Loewen NA. Review and meta-analysis of ab-interno trabeculectomy outcomes. Br J Ophthalmol. 2016 May;100(5):594-600. PMID: 26733487

2. Dang Y, Wang C, Shah P, Waxman S, Loewen RT, Hong Y, Esfandiari H, Loewen NA. Outflow enhancement by three different $a b$ interno trabeculectomy procedures in a porcine anterior segment model. Graefes Arch Clin Exp Ophthalmol [Internet]. 2018 May 2; Available from: http://dx.doi.org/10.1007/s00417-018-3990-0 PMID: 29721662

3. Vold S, Ahmed IIK, Craven ER, Mattox C, Stamper R, Packer M, Brown RH, lanchulev T, CyPass Study Group. TwoYear COMPASS Trial Results: Supraciliary Microstenting with Phacoemulsification in Patients with Open-Angle Glaucoma and Cataracts. Ophthalmology [Internet]. 2016 Aug 6; Available from: http://dx.doi.org/10.1016/j.ophtha.2016.06.032 PMID: 27506486

4. Capitena Young CE, Ammar DA, Seibold LK, Pantcheva MB, SooHoo JR, Kahook MY. Histopathologic Examination of Trabecular Meshwork Changes After Trabecular Bypass Stent Implantation. J Glaucoma. 2018 Jul;27(7):606609. PMID: 29965891

5. Shah M, Campos-Möller X, Werner L, Mamalis N, Ahmed IIK. Midterm failure of combined phacoemulsification with trabecular microbypass stenting: Clinicopathological analysis. J Cataract Refract Surg. Elsevier; 2018 May 1;44(5):654-657.

6. Khan M, Saheb H, Neelakantan A, Fellman R, Vest Z, Harasymowycz P, Ahmed IIK. Efficacy and safety of combined cataract surgery with 2 trabecular microbypass stents versus ab interno trabeculotomy. J Cataract Refract Surg. 2015 Aug;41(8):1716-1724. PMID: 26432130

7. Kurji K, Rudnisky CJ, Rayat JS, Arora S, Sandhu S, Damji KF, Dorey MW. Phaco-trabectome versus phaco-iStent in patients with open-angle glaucoma. Can J Ophthalmol. 2017 Feb;52(1):99-106. PMID: 28237158

8. lacus SM, King G, Porro G. Causal Inference without Balance Checking: Coarsened Exact Matching. Polit Anal. 2012 Jan 1;20(1):1-24.

9. Parikh HA, Bussel II, Schuman JS, Brown EN, Loewen NA. Coarsened Exact Matching of Phaco-Trabectome to Trabectome in Phakic Patients: Lack of Additional Pressure Reduction from Phacoemulsification. PLoS One. 2016 Feb 19;11(2):e0149384. PMCID: PMC4760733

10. Esfandiari H, Shazly T, Waxman S, Kola S, Kaplowitz KB, Brown EN, Loewen NA. Similar Performance of Trabectome and Ahmed Glaucoma Devices in a Propensity Score Matched Comparison. J Glaucoma [Internet]. 2018 Apr 2; Available from: http://dx.doi.org/10.1097/IJG.0000000000000960 PMID: 29613976

11. Kostanyan T, Shazly T, Kaplowitz KB, Wang SZ, Kola S, Brown EN, Loewen NA. Longer-term Baerveldt to Trabectome glaucoma surgery comparison using propensity score matching. Graefes Arch Clin Exp Ophthalmol [Internet]. 2017 Sep 25; Available from: http://dx.doi.org/10.1007/s00417-017-3804-9 PMID: 28944413

12. Dang $\mathrm{Y}$, Waxman S, Wang C, Shah P, Loewen RT, Loewen NA. Intraocular pressure elevation precedes a phagocytosis decline in a model of pigmentary glaucoma. F1000Res. 2018 Feb 12;7:174. PMCID: PMC5915754

13. Sherwood ME, Richardson TM. Phagocytosis by trabecular meshwork cells: sequence of events in cats and monkeys. Exp Eye Res. Elsevier; 1988 Jun;46(6):881-895. PMID: 3197758

14. Streilein JW, Masli S, Takeuchi M, Kezuka T. The eye's view of antigen presentation. Hum Immunol. 2002 
Jun;63(6):435-443. PMID: 12039518

15. Stein-Streilein J, Streilein JW. Anterior chamber associated immune deviation (ACAID): regulation, biological relevance, and implications for therapy. Int Rev Immunol. 2002 Mar;21(2-3):123-152. PMID: 12424840

16. Ng M, Sample PA, Pascual JP, Zangwill LM, Girkin CA, Liebmann JM, Weinreb RN, Racette L. Comparison of visual field severity classification systems for glaucoma. J Glaucoma. 2012 Oct;21(8):551-561. PMCID: PMC3246042

17. King G. Nearest Neighbor Matching [Internet]. [cited 2018 Jun 8]. Available from: https://r.iq.harvard.edu/docs/matchit/2.4-15/Nearest_Neighbor_Match.html

18. Fallano K, Bussel I, Kagemann L, Lathrop KL, Loewen NA. Training strategies and outcomes of $a b$ interno trabeculectomy with the trabectome. F1000Res. 2017 Jan 23;6:67. PMCID: PMC5428488

19. Kaplowitz K, Abazari A, Honkanen R, Loewen N. iStent surgery as an option for mild to moderate glaucoma. Expert Rev Ophthalmol. 2014;9(1):11-16.

20. Esfandiari $\mathrm{H}$, Hassanpour $\mathrm{K}$, Yaseri M, Loewen NA. Extended pharmacological miosis is superfluous after glaucoma angle surgery: A retrospective study. F1000Res [Internet]. 2018 Feb 12 [cited 2018 Feb 22];7. Available from: https://f1000research.com/articles/7-178/v1/pdf

21. Kaplowitz K, Loewen NA. Trabectome-Mediated Ab Interno Trabeculectomy for Secondary Glaucoma or as a Secondary Procedure. In: Aref AA, Varma R, editors. Advanced Glaucoma Surgery. Cham: Springer International Publishing; 2015. p. 15-29.

22. Parikh HA, Loewen RT, Roy P, Schuman JS, Lathrop KL, Loewen NA. Differential Canalograms Detect Outflow Changes from Trabecular Micro-Bypass Stents and Ab Interno Trabeculectomy. Sci Rep. 2016 Nov 4;6:34705. PMCID: PMC5095709

23. Wang Q, Harasymowycz P. Goniopuncture in the treatment of short-term post-Trabectome intraocular pressure elevation: a retrospective case series study. J Glaucoma. 2013 Oct;22(8):e17-20. PMID: 22828005

24. Bussel II, Kaplowitz K, Schuman JS, Loewen NA, Trabectome Study Group. Outcomes of ab interno trabeculectomy with the trabectome by degree of angle opening. Br J Ophthalmol. bjo.bmj.com; 2015 Jul;99(7):914-919. PMCID: PMC4501175

25. Sit AJ, McLaren JW. Measurement of episcleral venous pressure. Exp Eye Res. 2011 Sep;93(3):291-298. PMID: 21621536

26. McDonnell F, Dismuke WM, Overby DR, Stamer WD. Pharmacological regulation of outflow resistance distal to Schlemm's canal. Am J Physiol Cell Physiol. 2018 Jul 1;315(1):C44-C51. PMCID: PMC6087729

27. Waxman S, Wang C, Dang Y, Hong Y, Esfandiari H, Shah P, Lathrop KL, Loewen RT, Loewen NA. StructureFunction Changes of the Distal Outflow Tract in Response to Nitric Oxide. Invest Ophthalmol Vis Sci In Press [Internet]. [cited 2018 May 29]; Available from: https://www.preprints.org/manuscript/201806.0075/v1

28. Waxman S, Loewen RT, Dang Y, Watkins SC, Watson AM, Loewen NA. High-Resolution, Three-Dimensional Reconstruction of the Outflow Tract Demonstrates Segmental Differences in Cleared Eyes. Invest Ophthalmol Vis Sci. The Association for Research in Vision and Ophthalmology; 2018 May 1;59(6):2371-2380. PMCID: PMC5939687

29. Toth M, Bazeer S, Gazzard G. Retrospective review of pressure reducing effect of iStent and Trabectome procedures combined with cataract surgery. Acta Ophthalmol [Internet]. 2016 Oct 1;94. Available from: 
http://dx.doi.org/10.1111/j.1755-3768.2016.0466

30. Esfandiari H, Shah P, Torkian P, Conner IP, Schuman JS, Hassanpour K, Loewen NA. Five-year clinical outcomes of combined phacoemulsification and trabectome surgery at a single glaucoma center. Graefes Arch Clin Exp Ophthalmol [Internet]. 2018 Sep 26; Available from: http://dx.doi.org/10.1007/s00417-018-4146-y PMID: 30259089

31. Singh K, Lee BL, Wilson MR, Glaucoma Modified RAND-Like Methodology Group. A panel assessment of glaucoma management: modification of existing RAND-like methodology for consensus in ophthalmology. Part II: Results and interpretation. Am J Ophthalmol. 2008 Mar;145(3):575-581. PMID: 18191098

32. Pakravan M, Esfandiari H, Yazdani S, Douzandeh A, Amouhashemi N, Yaseri M, Pakravan P. Mitomycin Caugmented trabeculectomy: subtenon injection versus soaked sponges: a randomised clinical trial. $\mathrm{Br} \mathrm{J}$ Ophthalmol. 2017 Sep;101(9):1275-1280. PMID: 28100482

33. Prum BE Jr, Rosenberg LF, Gedde SJ, Mansberger SL, Stein JD, Moroi SE, Herndon LW Jr, Lim MC, Williams RD. Primary Open-Angle Glaucoma Preferred Practice Pattern $\left({ }^{\circledR}\right)$ Guidelines. Ophthalmology. 2016 Jan;123(1):P41P111. PMID: 26581556

34. Samuelson TW, Katz LJ, Wells JM, Duh Y-J, Giamporcaro JE, US iStent Study Group. Randomized evaluation of the trabecular micro-bypass stent with phacoemulsification in patients with glaucoma and cataract. Ophthalmology. Elsevier; 2011 Mar;118(3):459-467. PMID: 20828829

35. Malvankar-Mehta MS, lordanous Y, Chen YN, Wang WW, Patel SS, Costella J, Hutnik CML. iStent with Phacoemulsification versus Phacoemulsification Alone for Patients with Glaucoma and Cataract: A MetaAnalysis. PLoS One. 2015 Jul 6;10(7):e0131770. PMID: 26147908

36. Neiweem AE, Bussel II, Schuman JS, Brown EN, Loewen NA. Glaucoma Surgery Calculator: Limited Additive Effect of Phacoemulsification on Intraocular Pressure in Ab Interno Trabeculectomy. PLoS One. $2016 \mathrm{Apr}$ 14;11(4):e0153585. PMCID: PMC4831696

37. Shareef S, Sharma S. Target Placement of iStent Combined with Phacoemulsification. In: Rosenberg ED, Nattis AS, Nattis RJ, editors. Operative Dictations in Ophthalmology. Cham: Springer International Publishing; 2017. p. 215-224.

38. Gonnermann J, Bertelmann E, Pahlitzsch M, Maier-Wenzel A-KB, Torun N, Klamann MKJ. Contralateral eye comparison study in MICS \& MIGS: Trabectome ${ }^{\circledR}$ vs. iStent inject ${ }^{\circledR}$. Graefes Arch Clin Exp Ophthalmol. 2017 Feb;255(2):359-365. PMID: 27815624 


\section{Tables}

\section{Table 1}

Table 1: Demographics and preoperative characteristics after matching.

\begin{tabular}{|c|c|c|c|}
\hline & $\begin{array}{c}\text { AIT } \\
n=48\end{array}$ & $\begin{array}{l}\text { TMB } \\
n=48\end{array}$ & P-value* \\
\hline Age & $70 \pm 9$ & $75 \pm 8$ & $<0.01^{*}$ \\
\hline Gender & & & $0.06+$ \\
\hline Female & $22(46 \%)$ & $32(67 \%)$ & \\
\hline Male & $26(54 \%)$ & $16(33 \%)$ & \\
\hline Ethnicity & & & $<0.01 * *$ \\
\hline African American & $11(23 \%)$ & $1(2 \%)$ & \\
\hline Caucasian & 36 (75\%) & 45 (94\%) & \\
\hline Hispanic & $0(0 \%)$ & $2(4 \%)$ & \\
\hline Other & $1(2 \%)$ & $0(0 \%)$ & \\
\hline Type of glaucoma & & & $1^{* *}$ \\
\hline Primary open angle & $44(92 \%)$ & $44(92 \%)$ & \\
\hline Pseudoexfoliation & $1(2 \%)$ & $1(2 \%)$ & \\
\hline Angle closure & $1(2 \%)$ & $1(2 \%)$ & \\
\hline Normal tension & $2(4 \%)$ & $2(4 \%)$ & \\
\hline Visual field damage & & & $0.3^{* *}$ \\
\hline Mild & $20(42 \%)$ & $17(35 \%)$ & \\
\hline Moderate & $13(27 \%)$ & 17 (35\%) & \\
\hline Advanced & $12(25 \%)$ & $7(15 \%)$ & \\
\hline Phacoemulsification combined & 48 (100\%) & 48 (100\%) & \\
\hline
\end{tabular}

*based on T-test. +based on Chi-square test. ${ }^{* *}$ Based on Fisher exact test. 


\section{Table 2}

Table 2: IOP and number of medications after matching.

\begin{tabular}{|l|c|c|c|c|c|c|}
\hline & \multicolumn{2}{|c|}{ IOP } & \multicolumn{3}{c|}{ Medications } & \\
\hline Baseline & $15.3 \pm 3.1$ & $15.3 \pm 3.1$ & matched & $1.3 \pm 1.0$ & $1.3 \pm 1.0$ & matched \\
\hline 1 months & $12.4 \pm 3.5$ & $14.5 \pm 3.9$ & $0.01^{*}$ & $1.3 \pm 1.0$ & $0.6 \pm 0.8$ & $<0.01^{*}$ \\
\hline 3 months & $12.7 \pm 3.2$ & $13.8 \pm 2.9$ & 0.06 & $1.0 \pm 1.0$ & $0.7 \pm 0.9$ & 0.11 \\
\hline 6 months & $13.3 \pm 3.0$ & $13.6 \pm 3.1$ & 0.69 & $0.6 \pm 1.0$ & $0.8 \pm 1.0$ & 0.19 \\
\hline 12 months & $13.4 \pm 3.8$ & $14.3 \pm 2.6$ & $0.04^{*}$ & $0.7 \pm 1.1$ & $0.9 \pm 1.0$ & 0.19 \\
\hline 18 months & $14.1 \pm 3.7$ & $14.2 \pm 3.4$ & 0.69 & $0.6 \pm 0.9$ & $1.1 \pm 1.2$ & 0.21 \\
\hline 24 months & $13.9 \pm 3.3$ & $16.8 \pm 2.8$ & $0.04^{*}$ & $0.7 \pm 1.0$ & $1.7 \pm 1.2$ & $0.04 *$ \\
\hline
\end{tabular}

Mean \pm standard deviation (SD), *Wilcoxon test. 


\section{Supplementary Table 1}

Supplementary Table 1

\begin{tabular}{|l|c|c|c|}
\hline & $\begin{array}{c}\text { AIT } \\
\mathbf{n}=\mathbf{1 5 4}\end{array}$ & $\begin{array}{c}\text { TBS } \\
\mathbf{n = 1 1 0}\end{array}$ & P value \\
\hline Age & & & \\
\hline Mean \pm SD & $70 \pm 10$ & $74 \pm 10$ & $0.01^{*}$ \\
\hline Range & $(46,97)$ & $(41,92)$ & \\
\hline Gender & & & $0.11+$ \\
\hline Female & $86(56 \%)$ & $75(68 \%)$ & \\
\hline Male & $68(44 \%)$ & $35(32 \%)$ & \\
\hline Ethnicity & & & $0.01^{* *}$ \\
\hline African American & $46(30 \%)$ & $5(4 \%)$ & \\
\hline Asian & $2(1 \%)$ & $0(0 \%)$ & \\
\hline Caucasian & $102(66 \%)$ & $103(94 \%)$ & \\
\hline Hispanic & $0(0 \%)$ & $2(2 \%)$ & \\
\hline Other & $4(3 \%)$ & $0(0 \%)$ & \\
\hline Type of Glaucoma & & & $0.03 * *$ \\
\hline Primary open angle & $87(56 \%)$ & $83(75 \%)$ & \\
\hline Pseudoexfoliation Glaucoma & $18(12 \%)$ & $4(4 \%)$ & \\
\hline Angle Closure Glaucoma & $17(12 \%)$ & $8(7 \%)$ & \\
\hline Pigment Dispersion & $9(6 \%)$ & $1(1 \%)$ & \\
\hline Ocular Hypertension & $2(1 \%)$ & $5(5 \%)$ & \\
\hline Normal Tension Glaucoma & $10(6 \%)$ & $6(5 \%)$ & \\
\hline Other & $11(7 \%)$ & $3(3 \%)$ & \\
\hline Visual field & $56(36.3 \%)$ & $29(26.3 \%)$ & \\
\hline Mild & & & \\
\hline Moderate & & & \\
\hline Advanced & & & \\
\hline Prior Surgeries & & & \\
\hline & & & \\
\hline
\end{tabular}




\begin{tabular}{|l|l|l|} 
SLT & $5(3.2 \%)$ & $8(7.2 \%)$ \\
\hline
\end{tabular}

*based on T-test. 'based on Chi-square test. **Based on Fisher exact test. SLT: selective laser trabeculoplasty. 


\section{Supplementary Table 2}

Table 2: Unmatched IOP and number of medications.

\begin{tabular}{|c|c|c|c|c|c|c|}
\hline & \multicolumn{2}{|c|}{ IOP } & \multicolumn{3}{c|}{ Medications } & \\
\hline & AIT & TBS & P value* & AIT & TBS & P value* \\
\hline Baseline & $17.6 \pm 5.2$ & $15.5 \pm 4.1$ & $<0.01$ & $1.4 \pm 1.2$ & $1.4 \pm 1.0$ & 0.78 \\
\hline 1 month & $13.2 \pm 3.6$ & $14.2 \pm 3.8$ & 0.06 & $1.3 \pm 0.8$ & $0.7 \pm 0.8$ & $<0.01$ \\
\hline 3 months & $13.7 \pm 3.5$ & $13.5 \pm 2.9$ & 0.81 & $0.9 \pm 1.0$ & $0.7 \pm 0.9$ & 0.15 \\
\hline 6 months & $14.1 \pm 3.2$ & $13.6 \pm 3.0$ & 0.20 & $0.7 \pm 1.0$ & $0.8 \pm 1.0$ & 0.21 \\
\hline 12 months & $14.2 \pm 3.3$ & $13.9 \pm 2.8$ & 0.74 & $0.7 \pm 1.1$ & $0.9 \pm 1.0$ & 0.10 \\
\hline 18 months & $14.5 \pm 3.3$ & $14.4 \pm 3.6$ & 0.84 & $0.7 \pm 1.0$ & $0.9 \pm 1.2$ & 0.18 \\
\hline 24 months & $14.6 \pm 3.3$ & $16.8 \pm 3.2$ & 0.01 & $0.8 \pm 1.1$ & $1.1 \pm 1.2$ & 0.28 \\
\hline
\end{tabular}

*based on Wilcoxon test. IOP: intraocular pressure. 


\section{Figures}

\section{Figure 1}
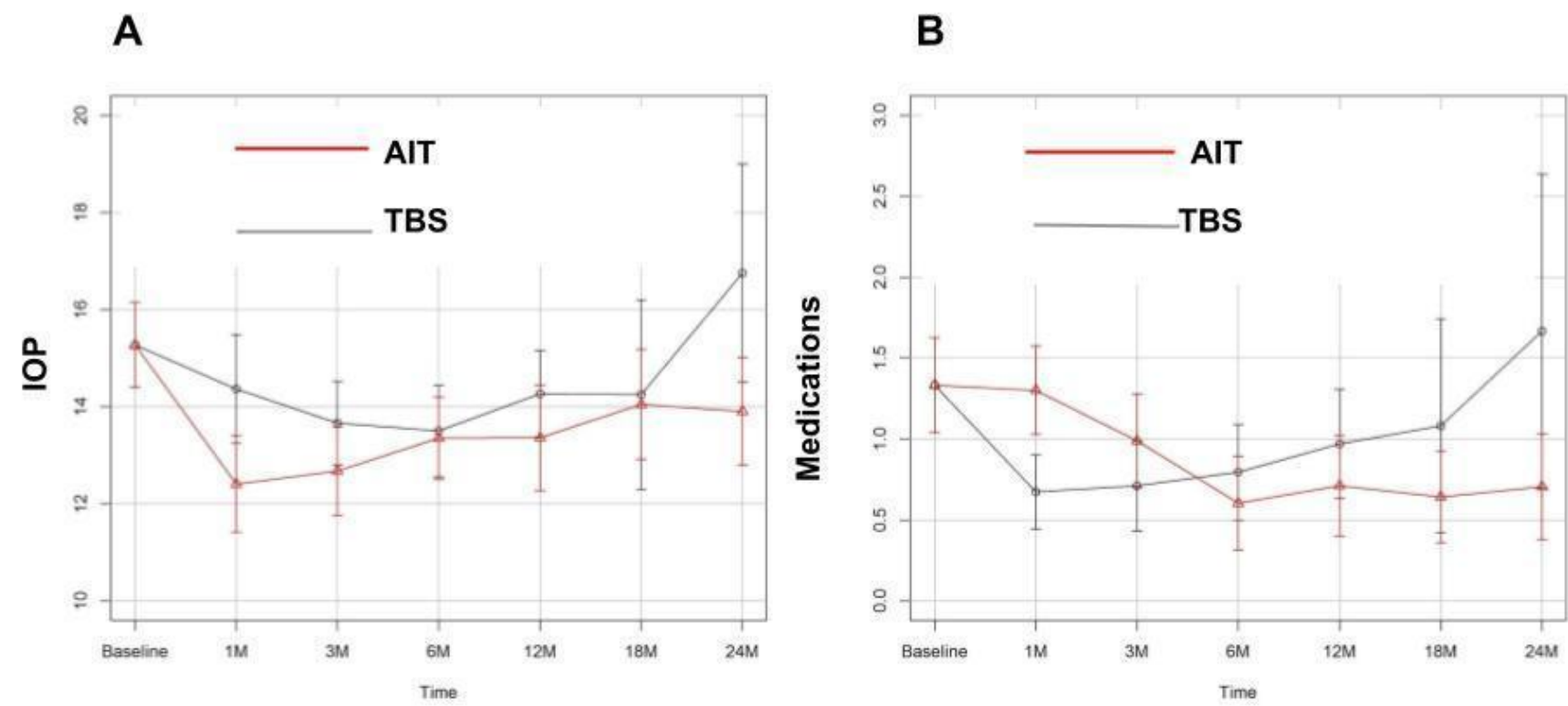

Figure 1: A) Intraocular pressure after Trabectome-mediated ab interno trabeculectomy (AIT) and TBS. The intraocular pressure (IOP, average \pm standard deviation) was significantly lower in AIT at month 1,12 , and 24 (all Ps <0.05). B) Glaucoma medications after AIT and iStent. The number of glaucoma medications (average \pm standard deviation (SD)) was significantly lower soon after the iStent but started to rise from 3 months up to final follow-up. 


\section{Figure 2}
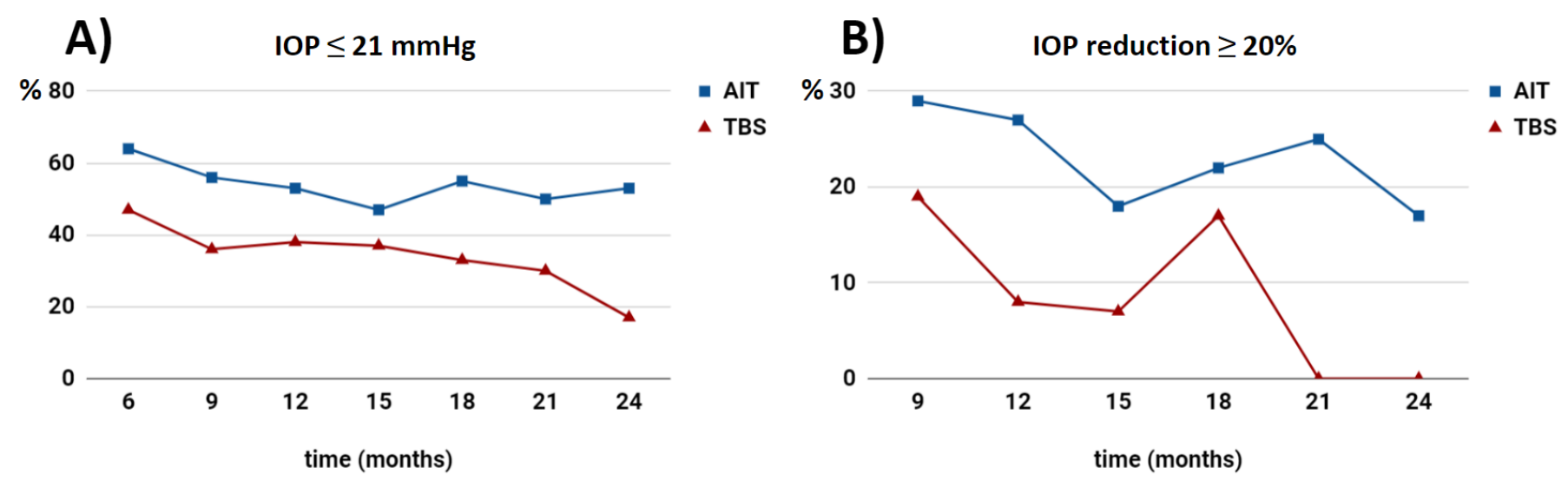

Figure 2: A) Proportion of patients with IOP $\leq 21 \mathrm{mmHg}$ without ocular hypotensive medications. B)

Proportion of patients with IOP reduction $\geq 20 \%$ without ocular hypotensive medications. 


\section{Figure 3}

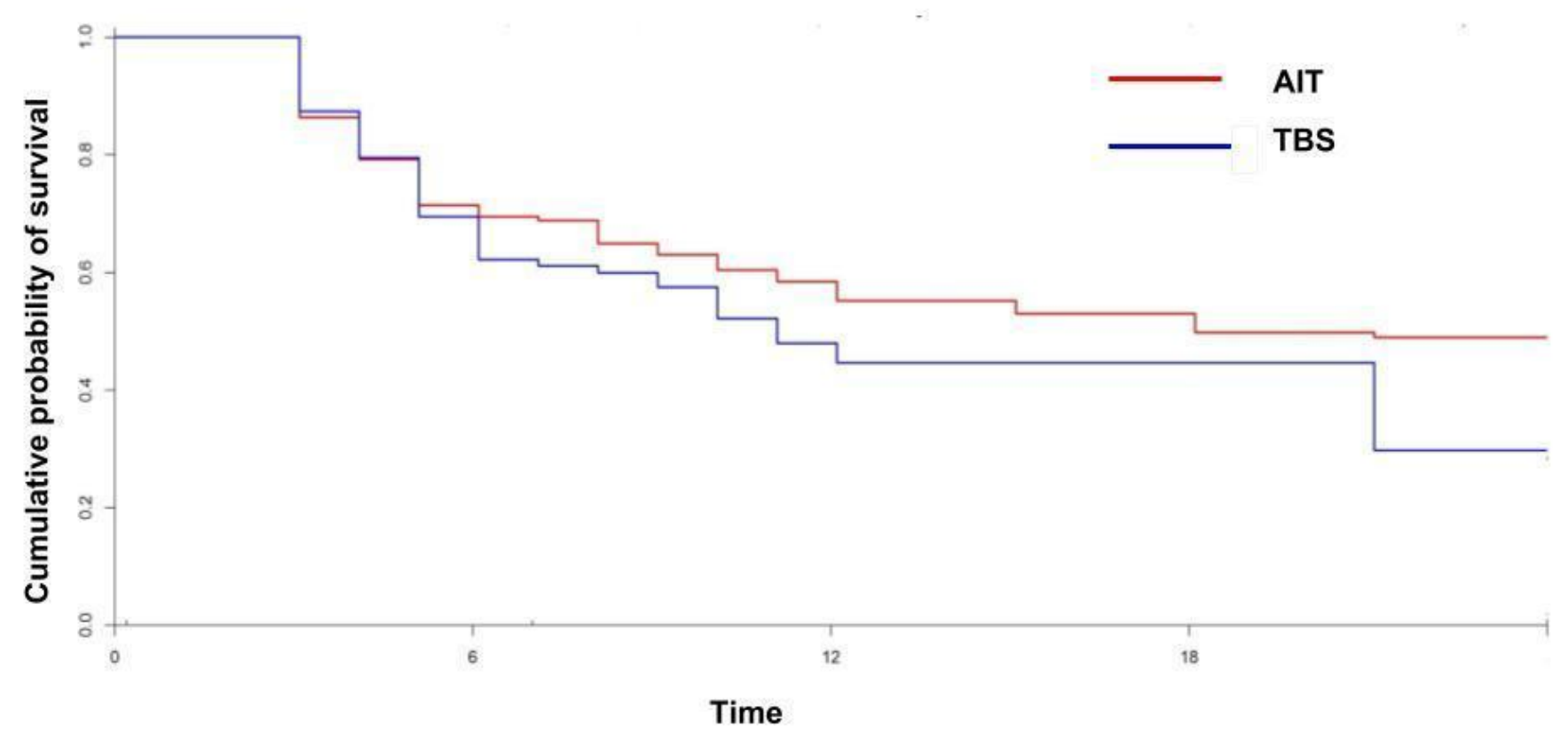

Figure 3: Kaplan-Meier survival plots for post-matched AIT and TBS with success defined as a final IOP of $\leq$ $21 \mathrm{mmHg}$ and a $20 \%$ reduction from baseline. While Success rates of AIT was higher at the final follow-up, the difference was not statistically significant (Log-Rank p-value=0.16). 


\section{Figure}

\section{Figure 4}

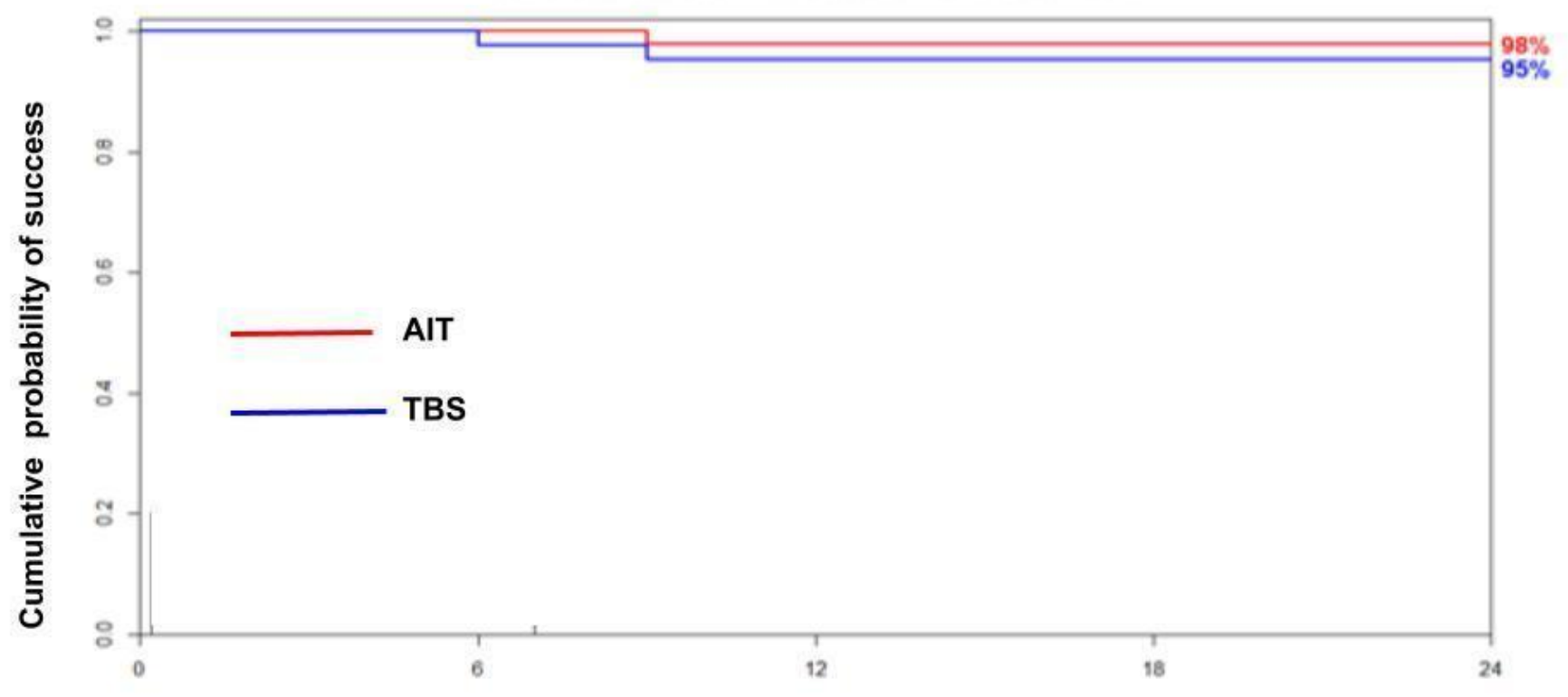

Time

Figure 4: Kaplan-Meier survival plots for post-matched AIT and TBS with success defined as a final IOP of $\leq$ $21 \mathrm{mmHg}$ or a $20 \%$ reduction from baseline. While Success rates of AIT was higher at the final follow-up, the difference was not statistically significant (Log- Rank p.value=0.31). 